\title{
Magnetic resonance imaging findings in acute myocarditis and correlation with immunohistological parameters
}

\author{
Rainer Röttgen • Robert Christiani • \\ Patrick Freyhardt • Matthias Gutberlet • \\ Hans Peter Schultheiss • Bernd Hamm • Uwe Kühl
}

Received: 9 May 2010 / Accepted: 25 October 2010 /Published online: 30 November 2010

(C) European Society of Radiology 2010

\begin{abstract}
Objective To evaluate the role of MRI in diagnosing acute myocarditis by correlation with immunohistological parameters.

Methods A total of 131 patients ( 85 men, 46 women; mean age, 44.9 years) with suspected acute myocarditis were examined by MRI. The relative water content of the left ventricular myocardium as well as relative and late enhancement was correlated with the immunohistological results in biopsy specimens.

Results Myocardial inflammation was confirmed by immunohistology in 82 of the 131 patients investigated and ruled out in 49 patients. The sensitivity, specificity and accuracy for diagnosing myocarditis in patients with immunohistologically proven disease were $48.8 \%, 73.8 \%$ and $57.3 \%$, respectively, for relative enhancement, $58.3 \%, 57.1 \%$ and $57.9 \%$ for relative water content, and $30.6 \%, 88.1 \%$ and $49.6 \%$ for late enhancement. A combination of all three
\end{abstract}

\footnotetext{
R. Röttgen $(\bowtie) \cdot$ R. Christiani $\cdot$ P. Freyhardt $\cdot$ B. Hamm Klinik für Strahlenheilkunde, Campus Virchow-Klinikum, Charite' Universitätsmedizin Berlin,

Augustenburger Platz 1,

13353, Berlin, Germany

e-mail: rainer.roettgen@charite.de

H. P. Schultheiss • U. Kühl

Klinik für Herz-, Kreislaufund Gefäßmedizin,

Campus Benjamin-Franklin, Charite' Universitätsmedizin Berlin, Hindenburgdamm 30,

12200, Berlin, Germany

\section{Gutberlet}

Herzzentrum Leipzig, Abteilung für diagnostische und interventionelle Radiologie,

Strümpelstrasse 39 ,

04289, Leipzig, Germany
}

parameters had 39,3\% sensitivity and 91,3\% specificity and $62,7 \%$ accuracy. Relative enhancement and late enhancement significantly correlated with the presence of myocarditis but relative oedema did not.

Conclusion Relative and late enhancement significantly correlate with the presence of myocarditis, while there is no significant correlation for relative oedema. Myocarditis cannot be reliably diagnosed using any of the three MRI parameters alone but combinations of parameters will improve specificity.

Keywords Myocarditis · Relative enhencement · Relative edema $\cdot$ Late enhancement $\cdot$ Immunhistology

\section{Introduction}

Magnetic resonance imaging (MRI) is playing an increasing role in suspected myocarditis, providing information on cardiac anatomy and function as well as typical signs of inflammation. These signs include an increase in tissue fluid or relative oedema [1-3], increased perfusion and vascular permeability of the myocardium at rest, reflected in the relative enhancement at MRI [1, 2, 4-7], and the loss of muscle cells and replacement with scar tissue, seen as late enhancement $[2,5,8-10]$. Together, the changes seen at MRI allow one to diagnose myocarditis and monitor its course. Nevertheless, our experience and published studies suggest that the role of MRI in general and the reliability of the different parameters for diagnosing inflammation are not well established. Having examined a relatively large group of patients by MRI for whom immunohistological findings in biopsy specimens are available to serve as an accurate indicator of the presence of myocardial inflammation, we performed a retrospective analysis in an attempt to 
create a sound basis for the diagnosis of myocarditis by MRI.

\section{Patients and methods}

Patients

The study included 131 patients. There were 85 men and 46 women ranging in age from 21 to 79 years with a mean age of $44.9 \pm 13.2$ years.

All patients had a preliminary diagnosis of myocarditis with a clinical indication for cardiac MRI and myocardial biopsy for immunohistological examination. The patients were included in the study only if MRI was performed within 2 weeks of the onset of clinical symptoms.

Follow-up MRI results were not included in the analysis; only the first MRI examination was analysed in patients who underwent serial MRI.

The study group of patients with proven acute myocarditis was defined by the presence of histologically and immunohistologically proven inflammatory infiltration; all other patients included served as controls.

\section{Materials and methods}

The diagnostic performance of MRI in confirming or ruling out myocarditis was evaluated by retrospectively analysing cardiovascular MRI and immunohistology of myocardial biopsies. We correlated the MRI findings with immunohistology because it is a more sensitive indicator of an inflammatory process than histological examinations, which are subject to a high sampling error.

For comparison of the two methods, the three features used for diagnosing myocarditis by MRI [6]-relative enhancement, relative water content and late enhancement, each measured during clinical routine MRI-correlated with the immunohistological findings in right ventricular myocardial biopsy specimens. According to the International Society and Federation of Cardiology classification, immunohistology is the more crucial than histology. According the Dallas-Classification the diagnosis of a myokarditis requires a inflammatory infiltration and a necroses of the myocytes. Additional the DallasClassification distinguish the borderline Myocarditis with few inflammatory cells and without a necroses of the myocytes. In our study we considerated the borderline myocarditis as a myocarditis.

\section{Magnetic resonance imaging}

All patients were examined on whole-body MRI with a field strength of 1.5 Tesla (Genesis Signa, General Electric Medical Systems, Milwaukee, WI, USA). The examinations were performed with two coils, a volume coil (body coil) and a surface coil (cardiac coil). Details of the sequences and techniques used are summarised in Table 1. Since the body coil has a rather homogeneous signal reception profile, this coil was used for measuring the relative signal intensities of myocardium and skeleton muscle. Thus, the inhomogeneous signal profile of the cardiac surface coil was avoided.

Table 1 Schedule with details of the sequences and techniques, which are used in this study

\begin{tabular}{llll}
\hline & Relative enhancement & Relative water content & Late enhancement \\
\hline MRI technique & FSE (fast spin echo) & STIR (short tau inversion recovery) & IRGE (inversion recovery gradient echo) \\
Sequence type & T1-weighted spin echo & T2-weighted triple inversion recovery & T1-weighted inversion recovery gradient echo \\
Coil & Body & Body & Cardiac \\
Contrast agent & Contrast-enhanced & Nonenhanced & Contrast-enhanced \\
Breathing & Normal breathing & Breath-hold & Breath-hold \\
Triggering & Prospective ECG triggering & Prospective ECG triggering & Prospective ECG triggering \\
TR (ms) & Depending on RR & Depending on RR & 5.5 \\
TE (ms) & 20 & 64 & 1.4 \\
IT (ms) & 150 & 140 & $180-240$ \\
ET & 32 & 32 & 1 \\
Matrix (pixels) & $256^{*} 256$ & $256^{*} 256$ & $256^{*} 192$ \\
FOV $(\mathrm{cm})$ & $38^{*} 38$ & $38^{*} 38$ & $35^{*} 35$ \\
SL (mm) & 20 & 20 & 8 \\
Spacing (mm) & 2 & 2 & 0 \\
NEX & 1 & 1 & 2
\end{tabular}

$T R$ time to repetition, $T E$ time to echo, $I T$ inversion time, $E T$ echotrain, FOV field of view, $S L$ slice thickness, $N E X$ number of excitations 
The analysis was performed in routine clinical fashion by two diagnostic radiologists in consensus; they each had extensive experience in cardiac MRI .

\section{Relative enhancement}

Relative enhancement [6] was calculated quantitatively from T1-weighted spin echo images obtained before and after intravenous administration of $0.1 \mathrm{mmol}$ of gadolinium-based contrast agent per kg body weight (Magnevist ${ }^{\circledR}$ Schering, Germany) with the injection-speed of $2 \mathrm{ml} / \mathrm{s}$. The time interval between injection and imaging was $15 \mathrm{~s}$. Here, myocardial signal intensities were calculated from five unenhanced axial slices and the corresponding five postcontrast slices. Two regions of interest (ROIs) were defined on unenhanced images: one ROI encompassing the left ventricular myocardium and another ROI in the musculus erector spinae (Fig. 1). The ROIs defined on the precontrast images were transferred $1: 1$ to the postcontrast images to ensure measurement of signal intensities in identical myocardial and skeletal muscle regions for calculation of relative enhancement. The resulting measurement values correspond to the signal intensities of the respective tissues modulated by differences in contrast medium uptake. Next, absolute enhancement was calculated for myocardium and skeletal muscle using the following formula:

absolute enhancement of the myocardial muscle $=\frac{\text { SI myocardial muscle with contrast }- \text { SI myocardial muscle native }}{\text { SI myocardial muscle native }}$

absolute enhancement of the skeleton muscle $=\frac{\text { SI the skeleton muscle with contrast }- \text { SI the skeleton muscle native }}{\text { SI l skeleton muscle native }}$

Finally, relative enhancement was calculated from absolute enhancement in cardiac and skeletal muscle as follows:

relative enhancement $=\frac{\text { absolute enhancement of the myocardial muscle }}{\text { absolute enhancement of the skeleton muscle }}$

Relative enhancement was calculated as the mean of the relative enhancements of all five slices. Based on current study data, the cut-off value for abnormal relative enhancement is 4 .

\section{Relative water content}

While relative enhancement is determined from axial images of the heart, we quantified the relative water content [6] or relative myocardial oedema ratio using three shortaxis slices perpendicular to the interventricular septum acquired with a fat-saturated T2-weighted triple-inversion recovery sequence. A circular ROI was defined encompassing the left ventricular myocardium in all three planes. Signal intensity of skeletal muscle was measured in the musculus erector spinae (Fig. 2). Drawing the ROI in skeletal muscle is limited by poor demarcation of the muscle from adjacent tissue on fat-saturated T2-weighted images; therefore, we used furthermore a T1-weighted sequence without fat supression in the same orientation to improve visualisation of the myocardium and skeletal muscle. The ROIs were transferred $1: 1$ to the corresponding $\mathrm{T} 2$-weighted sequences, allowing measurement of signal intensities in the anatomically correct positions. The measured signal intensities were used to calculate relative water content as follows:

relative water content $=\frac{\text { signal intensity of the myocardial muscle }}{\text { signal intensity of the skeleton muscle }}$

A mean value of relative water content was calculated from the values calculated for each of the three planes. Based on the results of earlier studies, relative water content $<2$ is considered normal, while values of 2 or greater are considered to indicate suspect findings.

Late enhancement

A contrast-enhanced T1-weighted inversion recovery gradient echo sequence was used to evaluate late enhancement [6]. Four-chamber, three-chamber and two- 


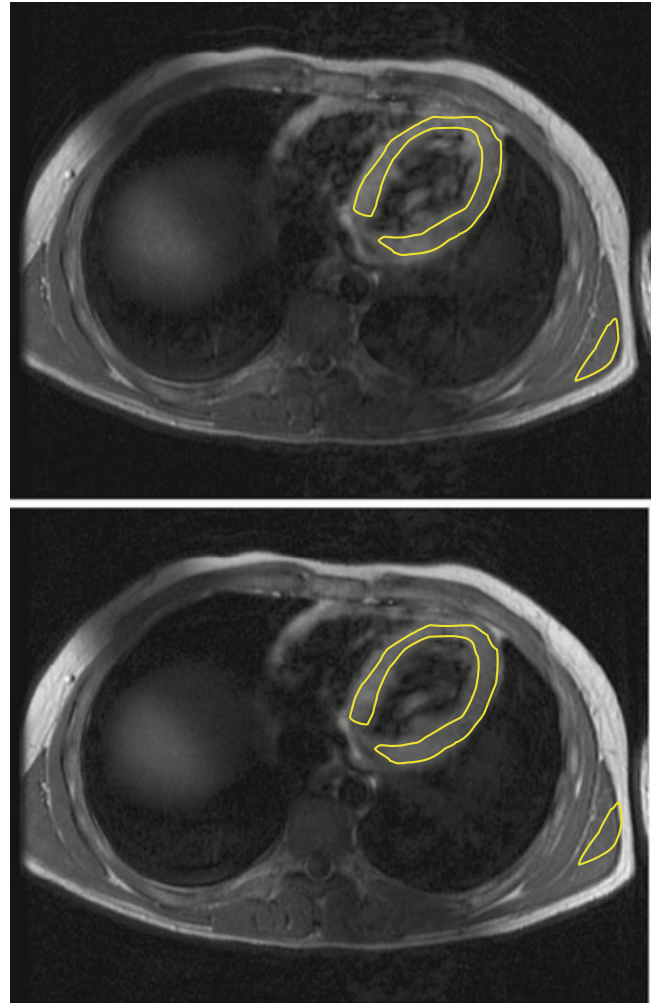

Fig. 1 Two axial slices before (top) and after (bottom) contrast medium administration. The yellow lines encircle typical ROIs used in our study. The latissimus dorsi muscle was used as reference

chamber views as well as $8-10$ short-axis views of the left ventricle were obtained to determine late enhancement. Images were acquired $20 \mathrm{~min}$ after contrast medium administration to capture persisting late enhancement, which is of interest here. Positive late enhancement is

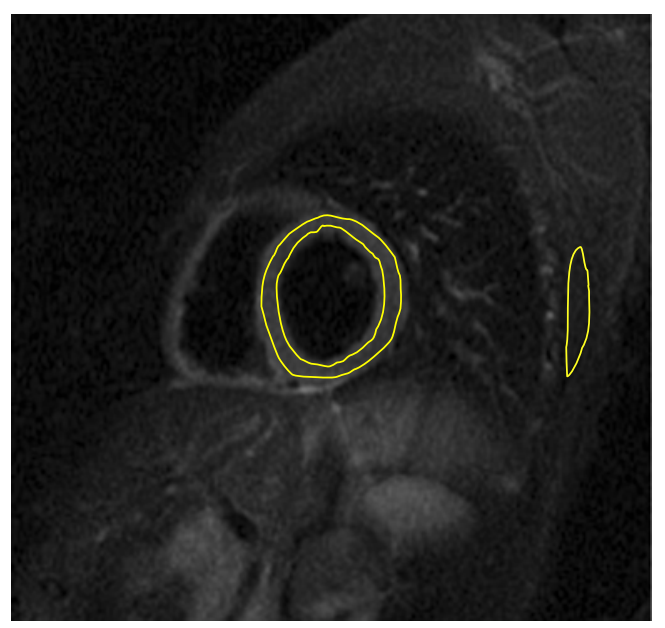

Fig. 2 Short-axis view perpendicular to the interventricular septum. The ROIs are outlined. Here, the pectoralis major muscle is used as reference. In this case we have measured a relative edema with the value: 2,4 characterised by an area of increased signal intensity (hyperintensity). That parameter was analyzed visually and not quantitatively (Fig. 3).

\section{Statistical analysis}

Dichotomous or non-metrical data were statistically analysed using the Chi-squared test according to Pearson, and results were verified using Fisher's exact test. In addition, logistic regression was used to identify correlations and calculate odds ratios predicting the likelihood of a variable affecting the result.

Metric results for relative enhancement and relative water content were presented in box-plot diagrams.

\section{Results}

Immunohistology confirmed myocardial inflammation in 82 of the 131 patients examined and excluded disease in 49 patients. The statistical results are summarised in Table 2.

\section{Relative enhancement}

There was a significant correlation between relative enhancement and immunohistological proof of myocarditis with $p=0.016$ (Pearson's Chi-squared test) or $p=0.02$ (Fisher's exact test). Mean relative enhancement was 4.4 with a standard deviation of \pm 2.17 for the subgroup of patients with proven myocarditis and 3.78 with a standard deviation of \pm 2.68 for those without proven disease (Fig. 4).

\section{Relative water content}

Our analysis revealed no significant correlation between relative water content and myocarditis. The results of the statistical analysis are presented in Table 2 and Fig. 5. We

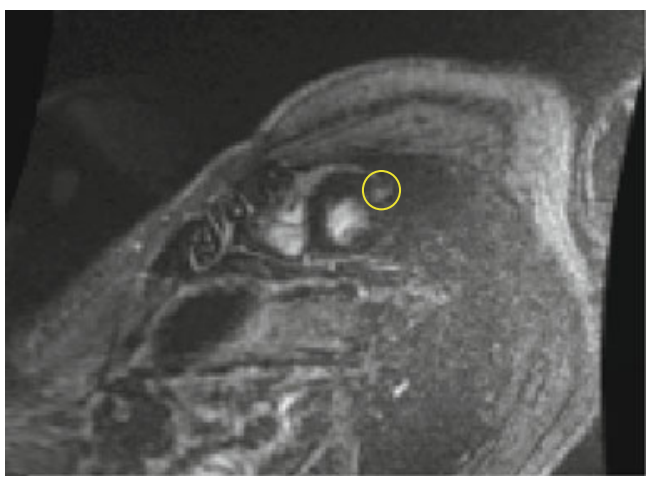

Fig. 3 Short-axis view of the left ventricle obtained $10 \mathrm{~min}$ after administration of contrast medium. There is an area of suspect subepicardial focal hyperintensity encircled by a yellow line 
Table 2 Comparison of our results with those oft wo recently published studies

\begin{tabular}{llcc}
\hline & Results of present study & $\begin{array}{c}\text { Gutberlet et al. Radiology } \\
\text { 2008 (9) }\end{array}$ & $\begin{array}{c}\text { Abdel- Aty et al. J Am Coll } \\
\text { Cardiol 2005 (3) }\end{array}$ \\
\hline Relative enhancement Sensitivity (\%) & 48.8 & 63 & 80 \\
Specificity (\%) & 73.8 & 86 & 68 \\
Positive predictive value (\%) & 78.4 & 86 & 74 \\
Negative predictive value (\%) & 42.5 & 63 & 75 \\
Accuracy (\%) & 57.3 & 72 & 74 \\
Relative water content Sensitivity (\%) & 58.3 & 67 & 84 \\
Specificity (\%) & 57.1 & 69 & 74 \\
Positive predictive value (\%) & 73.1 & 74 & 78 \\
Negative predictive value (\%) & 40.7 & 60 & 81 \\
Accuracy (\%) & 57.9 & 67 & 79 \\
Late enhancement Sensitivity (\%) & 30.6 & 27 & 44 \\
Specificity (\%) & 88.1 & 80 & 700 \\
Positive predictive value (\%) & 83.9 & 65 & 78 \\
Negative predictive value (\%) & 38.5 & 44 & 71 \\
Accuracy (\%) & 49.6 & 49 & \\
\hline
\end{tabular}

calculated the following values: $p=0.101$ (Pearson's Chisquared test) and $p=0.13$ (Fisher's exact test). Mean values and standard deviations were $2.02 \pm 0.34$ for patients with myocarditis and $1.99 \pm 0.48$ for patients without immunohistologically proven myocarditis.

Late enhancement

Our results show a significant correlation between late enhancement and myocarditis with the following values:

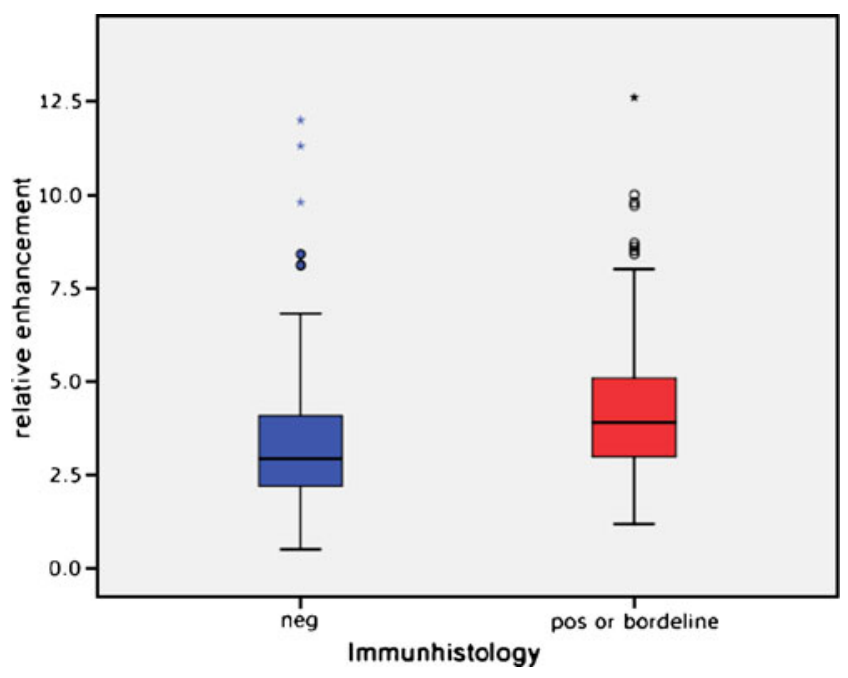

Fig. 4 Relative enhancement in the relationship with the results of immunohistology. Box-plot diagram illustrating the distribution of relative enhancement values in patients with negative immunohistology for myocarditis and those positive for the disease (including borderline myocarditis) $p=0.021$ (Pearson's Chi-squared test) and $p=0.027$ (Fisher's exact test). The results are summarised in Table 2.

In addition, the three parameters were evaluated in combination. In the logistic regression analysis, myocarditis was defined as a positive immunohistological test or a borderline positive test.

All parameters investigated showed significant differences $(p=0.05)$. The odds ratios for relative enhancement and late enhancement were 2.4 and 2.8, respectively, for the presence of myocarditis versus absence of myocarditis.

The sensitivity, specificity and accuracy calculated for the use of all three parameters - relative oedema, relative enhancement and late enhancement-were 39,3\%, 91,3\% and $62,7 \%$ respectively.

The results demonstrated significant differences with values of $p=0.013$ (Pearson's Chi-squared test) and $p=0.022$ (Fisher's exact test), which are below the assumed level of significance of $p=0.05$.

\section{Discussion}

\section{Relative enhancement}

The relative enhancement was calculated quantitatively from T1-weighted spin echo images obtained before and after intravenous administration of $0.1 \mathrm{mmol}$ of gadolinium-based contrast agent per $\mathrm{kg}$ body weight (Magnevist ${ }^{\circledR}$ Schering, Germany) with the injection-speed of $2 \mathrm{ml} / \mathrm{s}$. The time interval between injection and imaging was $15 \mathrm{~s}$. Here, myocardial signal intensities were calculated from five 


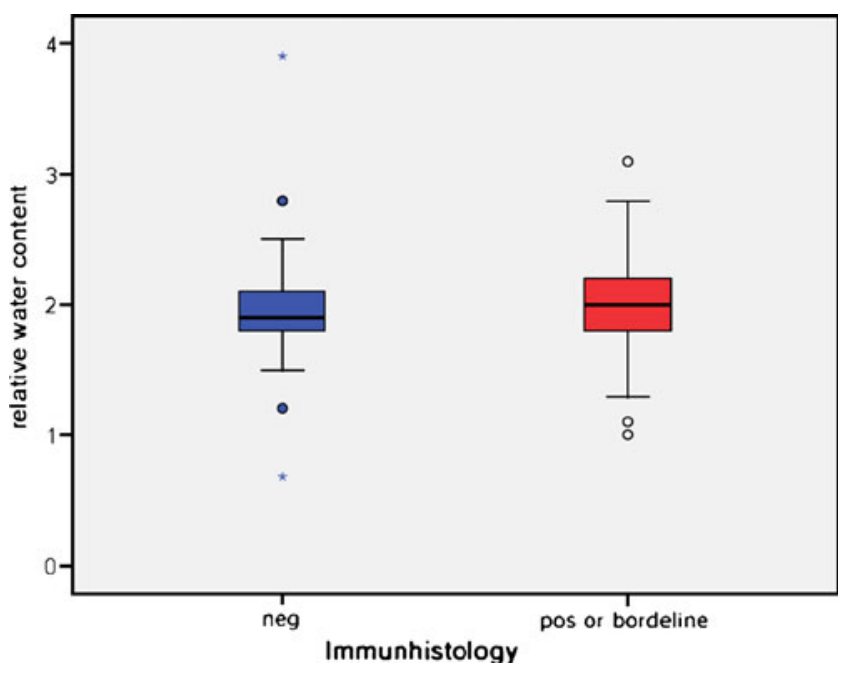

Fig. 5 Relative water content in the relation with the results of immunohistology. Box-plot diagram illustrating the distribution of relative water content in patients with negative immunohistology for myocarditis and those positive for the disease (including borderline myocarditis)

unenhanced axial slices and the corresponding five postcontrast slices. Two regions of interest (ROIs) were defined on unenhanced images: one ROI encompassing the left ventricular myocardium and another ROI in the musculus erector spinae

Since the body coil has a rather homogeneous signal reception profile, this coil was used for measuring the relative signal intensities of myocardium and skeleton muscle. Thus, the inhomogeneous signal profile of the cardiac surface coil was avoided.

In our study, relative enhancement has a sensitivity of only $48.8 \%$ for detecting immunohistologically proven acute myocarditis, which is low compared with data reported in the literature These results suggest that relative enhancement alone is not a reliable diagnostic criterion or screening test for acute myocarditis $[1,2]$. The negative predictive value and accuracy are also low at $42.5 \%$ and $42.5 \%$, respectively. On the other hand, specificity and positive predictive value are relatively high $(73.8 \%$ and $78.4 \%$, respectively) and similar to the values reported from comparable studies. Higher values were found by Gutberlet et al. [2]. One can only speculate why our results are poorer. One possible explanation is the difference in the number of patients examined: 131 in our study versus 83 in the study by Gutberlet et al. and 47 in the study by AbdelAty et al. [1]. These differences might lead to greater variance in the data and hence affect the results. The discrepancy may also be due to the fact that Gutberlet et al. only investigated patients with chronic myocarditis. It is conceivable that myocardial inflammation starting out as a focal lesion may extend throughout the myocardium as the condition becomes chronic. This assumption is confirmed by the results reported by Laissy et al. showing focal enhancement within the first 7 days after the onset of symptoms and diffuse relative enhancement further along the course [7]. As our patients were examined at the acute stage within 2 weeks of the onset of clinical symptoms, it is conceivable that the signal enhancement in an early focus of myocardial inflammation is compensated for by the normal signal intensity of surrounding healthy myocardial tissue. This is because we calculated a mean value of relative enhancement from the signal intensities measured throughout the myocardium visible on the respective images. This method may lead to a normal MRI result in patients with immunohistologically proven myocarditis. A third possible explanation is that different patient selection criteria were used, which might in particular explain the difference in results between our study and that of AbdelAty et al., who examined patients who potentially had myocarditis and completely healthy volunteers without symptoms. On the other hand, our entire study population consisted of individuals with a high clinical suspicion of acute myocarditis. As a result, our controls were patients who were immunohistologically negative for acute myocarditis. This means that we had no definitive evidence for the absence of myocarditis in the control patients as immunohistology has low sensitivity because of a relatively high sampling error of endomyocardial biopsy, which may explain the relatively moderate sensitivity of relative enhancement in our study. It is of note that in the study of Abdel-Aty et al. myocardial biopsies were obtained in only two patients, which might also have distorted the results because no gold standard diagnosis was available for most of the patients and definitive proof of myocarditis was not available for the patients with relative enhancement at MR [1].

In our study, the group of patients with immunohistologically proven myocarditis had a mean relative enhancement of 4.4 with a standard deviation of \pm 2.17 versus $3.78 \pm 2.68$ in the patients with negative immunohistology; the difference between the two groups is not significant. These values are comparable to those found by Gutberlet et al., whereas Abdel-Aty et al. reported a mean relative enhancement in diseased patients of 6.8 with a standard deviation of \pm 4.0 . The difference may be due to the small number of cases examined by Abdel-Aty et al. or the fact that they examined asymptomatic healthy individuals as controls. The latter point alone does not explain the higher mean value of relative enhancement as such but the examination of asymptomatic patients as controls might explain the clearer separation of the two groups in the study of Abdel-Aty et al. compared with a control group consisting of symptomatic patients with suspected myocarditis in our study. 
Logistic regression taking into account age and sex revealed no significant correlation between relative enhancement and myocarditis with $p=0.188$. The odds ratio is 2.4 ; in other words, there is a 2.4 -fold higher chance of relative enhancement being positive in patients with myocarditis versus patients without myocarditis. This is not a hard indicator but is meaningful as a clue in conjunction with other findings. In conclusion, relative enhancement alone is not a sufficient diagnostic parameter for myocarditis, and our results in acute myocarditis are even poorer than published data on chronic myocarditis [7]. Nevertheless, relative enhancement is useful as one of several criteria in diagnosing myocarditis.

\section{Relative water content}

The relative water content we found in our patients with proven myocarditis is below that reported by other authors $[1,2]$. Again, the discrepancies may be due to the problems outlined above (different numbers of patients, selection criteria, lack of proof of disease by endomyocardial biopsy); in addition, general problems associated with the measurement of water content may play a role. When there is oedema, the fluid content of tissue is increased. Most of the fluid comes from the blood vessels. Extravasation may be caused by inflammatory processes in the setting of myocarditis or might be due to inflammatory or destructive vasculopathies. Examples of vessel diseases include vasculitis or microangiopathy in diabetes mellitus. The results are also influenced by the fact that relative water content is calculated in relation to that of skeletal muscle. If there is additional oedema of the skeletal muscle of whatever aetiology, the MRI result for relative water content will be pseudonormal. Investigating the distribution of relative enhancement in myocardium and skeletal muscle in patients with myocarditis, Laissy et al. found increased enhancement of both the myocardium and peripheral skeletal muscle within the first 7 days after onset of symptoms, suggesting that there is either systemic inflammation or involvement of skeletal muscle [7]. This might also hold true for relative oedema.

In summary, relative water content alone is even less suitable for diagnosing myocarditis compared with relative enhancement. Relative water content may be interpreted as a clue pointing to the disease but, based on our results, this parameter does not allow more concrete diagnostic conclusions to be drawn.

Late enhancement

Late enhancement is the only one of the three MRI parameters for which we found a comparable or even higher statistical significance compared with the study of chronic myocarditis by Gutberlet et al. However, our results are again poorer than those reported by Abdel-Aty et al. Late enhancement has a high specificity compared with relative enhancement and relative water content, while sensitivity is low, rendering this parameter superior to the other two MRI criteria for confirming suspected disease with the low sensitivity precluding the use of this parameter for detecting myocarditis, e.g. in a screening test.

There are several possible explanations for the low sensitivity of late enhancement. Late enhancement, or the persistence of contrast agent in the interstitial space, occurs not only during acute inflammation but also when there is remodelling during healing or may reflect scar tissue or fibrosis as late sequelae of acute myocarditis [11]. It is conceivable that, despite symptomatic disease, the immunohistological examination may be positive before an organised focus of inflammation becomes morphologically apparent or before there is myocyte loss with subsequent scar formation or fibrosis, conditions that are associated with contrast medium extravasation into the interstitial space seen as late enhancement on MR images. Hence, it can be assumed that cell loss has not yet occurred in acute myocarditis and there is no necrosis or scar tissue to produce late enhancement. Late enhancement significantly correlates with immunohistologically proven myocarditis in our study ( $p=0.021$ or $p=0.027$ ). The odds ratio of approx. 2.8 suggests that the probability of seeing late enhancement in patients with myocarditis has the highest probability of all three MRI criteria investigated. Nevertheless, late enhancement alone does not provide a reliable diagnostic criterion for myocarditis.

Combining all three MRI parameters, a positive result for one of the three was found to have a sensitivity of $39,3 \%$ with the highest specificity of $91.3 \%$ being observed when all three criteria were positive.

Our results suggest that all MRI criteria should be used in combination to arrive at a well-founded conclusion regarding the presence of myocarditis, which is notoriously difficult to diagnose. A positive MRI result may thus serve as a basis for ordering further diagnostic tests. If all three MRI parameters are positive in a patient with the classical clinical symptoms, a diagnosis of myocarditis can be made with a high degree of specificity.

\section{Limitations}

In our study, endomyocardial biopsy specimens were sampled from different septal regions although some earlier publications already recommended targeted biopsy from potential sites of inflammation suggested by late enhance- 
ment on MR images [5, 12]. Non-targeted biopsy increases the sampling error, resulting in lower sensitivity [13]. Our biopsy technique may have distorted the MRI results as the immunohistological examination defined the group of patients with myocarditis in our study. The second problem directly following from this procedure is that we classified borderline myocarditis as positive for myocarditis. This milder form of disease may lead to less pronounced changes in the MRI parameters investigated, which also affects our results.

Although we investigated a larger number of patients than both Gutberlet et al. and Abdel-Aty et al., the study is still too small to obtain truly representative statistical data. This is suggested by the measure of quality of logistic regression, the Nagelkerke's R-square, which is 0.127 and thus well below the maximum possible value of nearly 1 .

While MRI is quite accurate, shown both in our study and the most important studies [14] reported in the literature, it should not be used alone to diagnose myocarditis. The final conclusion to be drawn from the results presented here is that all parameters that can be derived from cardiovascular magnetic resonance imaging taken together allow good non-invasive diagnosis of myocarditis with high specificity but have to be supplemented by additional tests for adequate diagnostic management of myocarditis.

\section{Conclusion}

The three parameters used for diagnosing acute myocarditis by MRI - relative oedema, relative enhancement and late enhancement - in part correlate significantly with the presence of myocarditis. Nevertheless, none of the three MRI parameters alone allows one to reliably confirm or rule out disease. The diagnosis can be made with a much higher degree of confidence when all three parameters are used, which specificity. But even the combination of MRI parameters does not allow detection of all cases of the disease, which is why the results should always be interpreted in conjunction with a patient's clinical symptoms, the findings of other diagnostic tests, and not least of all the results of myocardial biopsy.

Acknowledgements Concerning the immunohistological results in biopsy specimens this study was supported by a grant from Bayer Health
Care, Berlin, Germany Research Foundation (DFG), Transregional Collaborative Research Centre "Inflammatory Cardiomyopathy-Molecular Pathogenesis and Therapy” (SFB TR 1904 ).

\section{References}

1. Abdel-Aty H, Boye P, Zagrosek A et al (2005) Diagnostic performance of cardiovascular magnetic resonance in patients with suspected acute myocarditis. J Am Coll Cardiol 45:18151822

2. Gutberlet M, Spors B, Thoma T et al (2008) Suspected chronic myocarditis at cardiac MR: diagnostic accuracy and association with immunohistologically detected inflammation and viral persistence. Radiology 246:401-409

3. Zagrosek A, Abdel-Aty H, Boye P et al (2009) Cardiac magnetic resonance monitors reversible and irreversible myocardial injury in myocarditis. JACC Cardiovasc Imaging 2:131-138

4. Mangin M, Mahrholdt H, Sechtem U (2006) Diagnosis of myocarditis: description and assessment of available methods. Dtsch Med Wochenschr 131:1228-1234

5. Mahrholdt H, Goedecke C, Wagner A et al (2004) Cardiovascular magnetic resonance assessment of human myocarditis. Circulation 109:1250-1258

6. Friedrich M, Strohm O, Schulz-Menger J et al (1998) Contrast media-enhanced magnetic resonance imaging visualizes myocardial changes in the course of viral myocarditis. Circulation 97:1802-1809

7. Laissy JP, Messin B, Varenne O et al (2002) MRI of acute myocarditis - a comprehensive approach based on various imaging sequences. Chest 122:1638-1649

8. Gahide G, Bertrand D, Roubille R et al (2010) MR delayed enhancement imaging findings in suspected acute myocarditis. Eur Radiol 20:65-72

9. Korkusuz H, Esters P, Naguib N et al (2009) Acute myocarditis in a rat model: late gadolinium enhancement with histopathological correlation. Eur Radiol 19:2672-2678

10. Bohl S, Wassmuth R, Abdel-Aty H et al (2008) Delayed enhancement cardiac magnetic resonance imaging reveals typical patterns of myocardial injury in patients with various forms of non-ischemic heart disease. Int $\mathbf{J}$ Cardiovasc Imaging 24:597-607

11. Wu E, Judd RM, Vargas JD et al (2001) Visualisation of presence, location, and transmural extent of healed Q-wave and non-Qwave myocardial infarction. Lancet 357:21-28

12. Mangin M, Mahrholdt $\mathrm{H}$, Hager $\mathrm{H}$ et al (2005) Cardiovascular magnet resonance assessment of human myocarditis using a standardized combined right and left ventricular endomyocardial biopsy protocol. Circulation 112(Suppl 3):182

13. Hauck AJ, Kearney DL, Edwards WD (1989) Evaluation of postmortem endomyocardial biopsy specimens from 38 patients with lymphocytic myocarditis: implications for role of sampling error. Mayo Clin Proc 63:1235-1245

14. Friedrich MG, Sechtem U, Schulz-Menger J (2009) Cardiovascular magnetic resonance in myocarditis: A JACC white paper. J Am Coll Cardiol 53:1475-1487 